\title{
R\&D, Competition and Growth with Human Capital Accumulation Revisited
}

\author{
Dominique Bianco ${ }^{a}$
}

JEL-Classification: D43, J24, L16, 031, 041

Keywords: Endogenous growth, horizontal differentiation, technological change, imperfect competition

\section{Introduction}

BuccI (2003) studies the impact of competition in the intermediate goods sector on growth and the sectoral distribution of skills within an R\&D based growth model with human capital accumulation. By combining the LuCAs' (1988) model of human capital accumulation with the R\&D based model developed by Grossman and Helpman (1991) in the simplest possible way, BuCCI (2003) shows that the market power has a positive effect on growth.

Among the assumptions used by BUCCI (2003) to derive this result is that there is no difference between the intermediate goods share in final output, the returns to specialization and the degree of market power of monopolistic competitors. This leads to the natural question whether making such a difference to the model changes its predictions. In this note, we show that including this difference into the model developed by BuCCI (2003) eliminates the result mentioned above. Indeed, in this case, the imperfect competition has no effect on growth but rather a leveling effect.

a Department of Economics, University of Bourgogne and CNRS (LEG), 2 Boulevard Gabriel, BP 26611, F-21066 Dijon Cedex, France. I would like to thank, without implicating, J-L. Gaffard, C. Le Van and E. Salies for useful suggestions and comments on an earlier draft of this paper. The author also thanks Klaus Neusser (the editor) and anonymous referees for providing constructive comments and help in improving the contents of this paper. The usual disclaimer applies. Email: dominique.bianco@u-bourgogne.fr. 


\section{The Model}

The model developed is based on Bucci (2003). ${ }^{1}$ The economy is structured by three sectors: final goods sector, intermediate goods sector and R\&D sector. The final output sector produces output that can be used for consumption using human capital and intermediate goods. These are available in $n$ varieties and are produced by employing only human capital. The R\&D sector creates the blueprints for new varieties of intermediate goods which are produced by employing human capital. These blueprints are sold to the intermediate goods sector. Unlike the traditional R\&D based growth models, we assume that the supply of human capital may grow over time.

\subsection{The Final Goods Sector}

In this sector atomistic producers engage in perfect competition. The final goods sector produces a composite good $Y$ by using all the $j^{\text {th }}$ type of intermediate goods $x_{j}$ and human capital $H_{Y}{ }^{2}$ Production is given by:

$$
Y=A H_{Y}^{1-\lambda} n^{\gamma-\lambda\left(\frac{1}{\alpha}-1\right)}\left[\int_{0}^{n} x_{j}^{\alpha} d j\right]^{\frac{\lambda}{\alpha}}
$$

where $\alpha, \lambda, \gamma \in] 0,1[$ and $A$ are technological parameters. This production function allows us to disentangle the degree of market power of monopolistic competitors in the intermediate sector $1 / \alpha$, the intermediate goods share in final output $\lambda$ and the degree of returns from specialization $\gamma$. In this sense, this model is a generalization of Bucci's (2003) model. ${ }^{3}$ If we normalize to one the price of the final good, the profit of the representative firm is given by:

$$
\pi_{Y}=A H_{Y}^{1-\lambda} n^{\gamma-\lambda\left(\frac{1}{\alpha}-1\right)}\left[\int_{0}^{n} x_{j}^{\alpha} d j\right]^{\frac{\lambda}{\alpha}}-\int_{0}^{n} p_{j} x_{j} d j-w_{Y} H_{Y},
$$

1 We use Bucci's (2003) notation in order to have a direct comparison with his model.

2 Time subscripts are omitted whenever there is no risk of ambiguity.

3 Indeed, we obtain the Bucci's (2003) model by introducing the following constraints $\lambda=\alpha$, $\gamma=1-\alpha$. We can also note that the Bianco's (2009) model is also a particular case of our model $(\lambda=\alpha)$. For a comparison and a discussion about these two models, see Bianco (2009). 
where $w_{Y}$ is the wage rate in the final goods sector and $p_{j}$ is the price of the $j^{\text {th }}$ intermediate good. Under perfect competition in the final output market, the representative firm chooses intermediate goods and human capital in order to maximize its profit, taking prices as given and subject to its technological constraint. The first order conditions are:

$$
\begin{aligned}
& \frac{\partial \pi_{Y}}{\partial x_{j}}=\lambda A H_{Y}^{1-\lambda} n^{\gamma-\lambda\left(\frac{1}{\alpha}-1\right)} x_{j}^{\alpha-1}\left[\int_{0}^{n} x_{j}^{\alpha} d j\right]^{\frac{\lambda}{\alpha}-1}-p_{j}=0, \\
& \frac{\partial \pi_{Y}}{\partial H_{Y}}=(1-\lambda) A H_{Y}^{-\lambda} n^{\gamma-\lambda\left(\frac{1}{\alpha}-1\right)}\left[\int_{0}^{n} x_{j}^{\alpha} d j\right]^{\frac{\lambda}{\alpha}}-w_{Y}=0 .
\end{aligned}
$$

Equation (2) is the inverse demand function for the firm that produces the $j^{\text {th }}$ intermediate good whereas equation (3) characterizes the demand function of human capital.

\subsection{The Intermediate Goods Sector}

In the intermediate goods sector, producers engage in monopolistic competition. Each firm produces one horizontally differentiated intermediate good and has to buy a patented design before producing it. Following Grossman and Helpman (1991) and BuCCI (2003), we assume that each local intermediate monopolist has access to the same technology employing only human capital $h_{j}$ :

$$
x_{j}=B h_{j},
$$

where $B$ measures the productivity of human capital employed in this sector. We suppose that the behavior of firms which produce intermediate goods is governed by the principle of profit maximization at given factor prices under a technological constraint. The profit function of firms is as follows:

$$
\pi_{j}=p_{j} x_{j}-w h_{j}
$$

where $w$ is wage rate in the intermediate goods. Using the first order condition, we obtain the price of the $j^{\text {th }}$ intermediate good:

$$
p_{j}=\frac{w}{\alpha B}
$$


At the symmetric equilibrium, all the firms produce the same quantity of the intermediate good, face the same wage rate and by consequence fix the same price for their production. The price is equal to a constant mark up $1 / \alpha$ over the marginal cost $w / B$. Defining by

$$
H_{j}=\int_{0}^{n} h_{j} d j
$$

the total amount of human capital employed in the intermediate goods sector and assuming symmetry among intermediate goods producers, we can rewrite the equation (4) as follows:

$$
x_{j}=\frac{B H_{j}}{n} .
$$

Finally, the profit function of the firm which produces the $j^{\text {th }}$ intermediate good is:

$$
\pi_{j}=A \lambda(1-\alpha) B^{\lambda} n^{\gamma-1} H_{j}^{\lambda} H_{Y}^{1-\lambda} .
$$

\subsection{The R\&D Sector}

There are competitive research firms undertaking R\&D. Following BUCCI (2003), we assume that new blueprints are produced using an amount of human capital $H_{n}$ :

$$
\dot{n}=\mathrm{CH}_{n} \text {, }
$$

where $C>0$ represents the productivity of the R\&D process. Because of the perfect competition in the R\&D sector, we can obtain the real wage in this sector as a function of the profit flows associated to the latest intermediate in using the zero profit condition:

$$
w_{n} H_{n}=\dot{n} V_{n},
$$

where $w_{n}$ represents the real wage earned by human capital. $V_{n}$ is the real value of such a blueprint which is equal to :

$$
V_{n}=\int_{t}^{\infty} \pi_{j} e^{-r(\tau-t)} d \tau, \tau>t
$$


where $r$ is the real interest rate. Given $V_{n}$, the free entry condition leads to:

$$
w_{n}=C V_{n}
$$

\subsection{The Consumer Behavior}

The demand side is characterized by the representative household who holds assets in the form of ownership claims on firms and chooses plans for consumption $c$, asset holdings $a$ and human capital $h{ }^{4}$ Following LuCAs (1988), we assume that the household is endowed with one unit of time and optimally allocates a fraction $u$ of this time endowment to productive activities (final goods, intermediate goods and research production) and the remaining fraction $1-u$ to nonproductive activities (education). Following Romer (1990), we assume that the utility function of this consumer is ${ }^{5}$ :

$$
U=\int_{0}^{\infty} e^{-\rho t} \frac{c^{1-\theta}-1}{1-\theta} d t
$$

where $c$ is private consumption, $\rho>0$ is the rate of pure time preference and $\sigma=1 / \theta$ is the intertemporal elasticity of substitution. The flow budget constraint for the household is:

$$
\dot{a}=w u h+r a-c,
$$

where $w$ is the wage rate per unit of human capital services. The human capital supply function is given by:

$$
\dot{h}=\delta(1-u) h,
$$

where $\delta>0$ is a parameter reflecting the productivity of the education technology. From the maximization program of the consumer, ${ }^{6}$ we obtain the Euler equation:

$$
g_{c}=\frac{r-\rho}{\theta} .
$$

4 Like BucCI (2003), for the sake of simplicity, we assume zero population growth.

5 BuCCI (2003) uses a utility function which is logarithmic whereas the utility function used in this paper is more general.

6 The control variables of this problem are $c$ and $u$ whereas $a$ and $h$ are the state variables. $\epsilon_{1}$ and $\epsilon_{2}$ denote the shadow price of the household's asset holdings and human capital stock. 


\section{Equilibrium and the Steady State}

In this section, we characterize the equilibrium and give some analytic characterization of a balanced growth path.

\subsection{The Equilibrium}

It is now possible to characterize the human capital market equilibrium in the economy considered. In this market, because of the homogeneity and the perfect mobility across sectors, the arbitrage ensures that the wage rate that is earned by employees who work in the final goods, intermediate goods or $\mathrm{R} \& \mathrm{D}$ sectors is equal. As a result, the following two conditions must simultaneously be verified:

$$
\begin{gathered}
u^{*} H=H_{Y}+H_{j}+H_{n}, \\
w=w_{Y}=w_{n} .
\end{gathered}
$$

Equation (14) is a resource constraint, saying that at any point in the time the sum of the human capital demands coming from each activity must be equal to the total available supply. Equation (15) states that the wage earned by one unit of human capital is the same irrespective of the sector in which that unit of human capital is actually employed.

We can characterize the product market equilibrium in the economy considered. Indeed, in this market, the firms produce a final good which can be consumed. Consequently, the following condition must be verified :

$$
Y=C \text {. }
$$

Equation (16) is a resource constraint on the final goods sector.

We can describe the capital market equilibrium in our economy. Because the total value of the household's assets must be equal to the total value of firms, the following condition must be verified:

$$
a=n V_{n},
$$

where $V_{n}$ is given by the equation (9) and satisfies the following asset pricing equation:

$$
V_{n}=r V_{n}-\pi_{j}
$$




\subsection{The Steady State}

At the steady state, all variables as $Y, c, n, a, H^{7} H_{Y}, H_{j}, H_{n}$ grow at a positive constant rate.

Proposition 1: If $u$ is constant, all the other variables grow at strictly positive rates with :

$$
\begin{aligned}
& g_{H}=g_{H_{Y}}=g_{H_{j}}=g_{H_{n}}=g_{n}, \\
& g_{Y}=g_{c}=g_{a}=(\gamma+1) g_{n} .
\end{aligned}
$$

Proof. From the equilibrium on the human capital market, given by the equation (14), it easy to show that $g_{H}=g_{H_{Y}}=g_{H_{j}}=g_{H_{n}}$ if $u$ is constant. From the definition of the firm research process, given by the equation (8), we obtain that $g_{n}=g_{H_{n}}$. Now, if we combine the two last equations, we obtain the equation (18). From the equilibrium on the product market, given by the equation (16), it easy to find that $g_{Y}=g_{c}$. The equation (17) implies that $g_{a}=g_{n}+g_{V_{n}}$. By substituting equation (6) into equation (1), then by log-differentiating the equation (1), we obtain $g_{Y}=(\gamma+1) g_{n}$. By combining the previous equations, we arrive at the equation (19).

Using the previous equations, we can demonstrate the following steady state equilibrium values ${ }^{8}$ for the relevant variables of the model ${ }^{9}$ :

$$
\begin{aligned}
& u^{*}=\frac{(\gamma+1) \delta(\theta-1)+\rho}{\delta(\gamma(\theta-1)+\theta)}, \\
& g_{n}=\frac{\delta-\rho}{\gamma(\theta-1)+\theta}, \\
& g_{Y}=\frac{(\gamma+1)(\delta-\rho)}{\gamma(\theta-1)+\theta} .
\end{aligned}
$$

7 Given the assumptions on the size of the representative household and the population growth rate, $h \equiv H$ which implies that we can use $g_{H}$ instead of $g_{h}$.

8 For a detailed demonstration, see the appendix.

9 We assume that $\theta>1$ and $\delta>\rho$ in order to have all variables growing at a positive growth rate. 
The equation (20) represents the optimal and constant fraction of the household's time endowment that it will decide to devote to work $\left(u^{*}\right)$ in equilibrium. Equation (21) states that the growth rate of human capital and the innovation activity are equal and depend on technological $(\delta, \gamma)$ and preference parameters $(\theta, \rho)$. Equation (22) shows that the growth rate is a function of technological $(\delta, \gamma)$ and preference parameters $(\theta, \rho)$.

First of all, unlike Lucas (1988) and Blackburn, Hung, and Pozzolo (2000), growth is not only driven by human capital accumulation $\left(g_{h}\right)$ but also by the technology used by firms in the final goods sector $(\gamma)$. Secondly, like Bucci (2003), Bianco (2009) and Blackburn, Hung, and Pozzolo (2000), the competition between intermediate sector producers does not play any role in the consumer's decision about how much time to invest in education $\left(u^{*}\right)$. Thirdly, unlike BuCCI (2003) and Bianco (2009), the competition has no effect on growth.

\section{The Relationship between Product Market Competition and Growth}

In this section, we study the long run relationship between competition and growth in the model presented above. Following most authors, we use the so-called Lerner Index to gauge the intensity of market power within a market. Such an index is defined by the ratio of price $P$ minus marginal cost $C m$ over price. Using the definition of a mark-up Markup $=P / C m$ and Lerner Index LernerIndex $=(P-C m) / P$, we can use (5) to define a proxy of competition ${ }^{10}$ as follows:

$$
1-\text { LernerIndex }=\alpha .
$$

Following Alvarez-Pelaez and Groth (2005), we disentangle the monopolistic mark-up in the intermediate goods sector, the intermediate goods share in the final output and the returns to specialization in order to have a better measurement of competition. Indeed, in our simple generalization of Bucci's (2003) model, the competition is completely independent of the intermediate goods share in the final output and the returns to specialization. In this framework, the competition has no effect on growth.

10 This is the same measure of product market competition used by BIANCO (2009 among others. BuCCI (2003) does not use the same definition of competition. Indeed, he prefers to use the power market defined by $1 / \alpha$. However, since these two definitions are inversely related, our results are not affected by the definition used. 
Proposition 2: The competition has no effect on growth for all positive values of $\rho, \eta, L$ and $\gamma, \lambda \in] 0,1[$.

Proof. The proof is obtained by differentiating (22) with respect to $\alpha$ :

$$
\frac{\partial g_{Y}}{\partial \alpha}=0
$$

In Bucci's (2003) model, the author shows that the competition has a positive effect on growth. This result is due to the specification of the production function in which the market power is linked to the returns to specialization and the intermediate goods share in final output. Indeed, if we disentangle these three parameters, we show that the competition has no effect on growth. Only the returns to specialization and preference parameters have an impact on growth. This results is explained by the complementarity between these two sources of growth: innovation and human capital. Indeed, this complementarity means that the growth of innovation activity is induced by the growth of human capital stock which is independent of competition.

Nevertheless, we can show that the competition has a positive effect on the level of production. Since all the production is consumed, this means that an increase in competition affects consumer's welfare positively.

In order to compute the equilibrium shares of human capital devoted respectively to research $\left(s_{n}\right)$, intermediate goods sector $\left(s_{j}\right)$, consumption goods sector $\left(s_{y}\right)$ and human capital accumulation $\left(s_{b}\right)$, we have first to determine an expression for the ratio of equilibrium human capital to technological capital $(R \equiv H / n)$. To do so, we use equations $(14,64,65$ and 66$)$, we obtain:

$$
\frac{H_{n}}{n}=\frac{\delta+\frac{\delta}{\lambda(\alpha-1)}}{C}+\frac{R((1+\gamma) \delta(\theta-1)+\rho)}{\delta(\gamma(\theta-1)+\theta)} .
$$

Using the equations $(8,23$ and 62$)$, we obtain:

$$
R \equiv \frac{H}{n}=\frac{\delta\left(\frac{\delta(\gamma-(\gamma-1) \theta)}{(\alpha-1) \lambda((\gamma+1) \delta(\theta-1)+\rho)}-1\right)}{C}
$$


In equation (24), the ratio of human to technological capital $(R)$ is determined by preference $(\theta, \rho)$ and technological parameters $(C, \delta, \lambda, \alpha, \gamma)$ of the model.

Given $R$, the shares of human capital devoted to each sector employing this factor of production are easily determined:

$$
\begin{aligned}
& s_{j}=\frac{\alpha \lambda((\gamma+1) \delta(\theta-1)+\rho)}{\delta((\alpha-1)(\gamma+1)(\theta-1) \lambda+\gamma \theta-\gamma+\theta)+(\alpha-1) \lambda \rho}, \\
& s_{Y}=\frac{(\lambda-1)((-\gamma-1) \delta(\theta-1)-\rho)}{\delta((\alpha-1)(\gamma+1)(\theta-1) \lambda+\gamma \theta-\gamma+\theta)+(\alpha-1) \lambda \rho}, \\
& s_{n}=\frac{\delta-\rho}{\delta(\gamma(\theta-1)+\theta)\left(\frac{\delta(\gamma-(\gamma+1) \theta)}{(\alpha-1) \lambda((\gamma+1) \delta(\theta-1)+\rho)}-1\right)}, \\
& s_{H}=\frac{\delta-\rho}{\delta(\gamma(\theta-1)+\theta)} .
\end{aligned}
$$

As in Bucci's (2003) model, the equilibrium distribution of human capital stock across economic activities is influenced by the productivity of education technology $(\delta)$, the subjective discount rate $(\rho)$ and the level of product market competition in the intermediate sector $(\alpha)$. Unlike BucCI (2003), in our model, these stocks of human capital are also determined by the preference parameter $(\theta)$, the degree of returns from specialization $(\gamma)$ and the intermediate goods share in final output $(\lambda)$.

Proposition 3. Competition has a positive effect on the level of production per capita for all positive values of $\rho, \eta, L$ and $\gamma, \lambda \in] 0,1[$.

Proof. Using the production function (equation (1)), the quantity of the $j^{\text {th }}$ intermediate good (equation(6)) and dividing the output $(Y)$ by worker $(H)$, we get production per capita $(y)$ :

$$
y=A B^{\lambda} n^{\gamma} s_{Y}^{1-\lambda} s_{j}^{\lambda}
$$

We can rewrite this equation as follows :

$$
y \equiv F\left(s_{Y}(\alpha), s_{j}(\alpha)\right)
$$


The derivative of this equation is:

$$
\frac{\partial y}{\partial \alpha}=\frac{\partial F}{\partial s_{Y}} \frac{\partial s_{Y}}{\partial \alpha}+\frac{\partial F}{\partial s_{j}} \frac{\partial s_{j}}{\partial \alpha}
$$

Thus, $\partial y / \partial \alpha>0$ if and only if :

$$
\frac{\frac{\partial F}{\partial s_{j}}}{\frac{\partial F}{\partial s_{Y}}}>-\frac{\frac{\partial s_{Y}}{\partial \alpha}}{\frac{\partial s_{j}}{\partial \alpha}} .
$$

Or, we have:

$$
\begin{aligned}
\frac{\partial F}{\partial s_{Y}} & =(1-\lambda) A B^{\lambda} n^{\gamma} s_{Y}^{-\lambda} s_{j}^{\lambda}, \\
\frac{\partial F}{\partial s_{j}} & =\lambda A B^{\lambda} n^{\gamma} s_{Y}^{1-\lambda} s_{j}^{\lambda-1}, \\
\frac{\partial s_{Y}}{\partial \alpha} & =\frac{(\lambda-1) \lambda((\gamma+1) \delta(\theta-1)+\rho)^{2}}{(\delta((\alpha-1)(\gamma+1)(\theta-1) \lambda+\gamma \theta-\gamma+\theta)+(\alpha-1) \lambda \rho)^{2}}, \\
\frac{\partial s_{j}}{\partial \alpha} & =-\frac{\lambda((\gamma+1) \delta(\theta-1)+\rho)(\delta((\gamma+1)(\theta-1) \lambda+\gamma(-\theta)+\gamma-\theta)+\lambda \rho)}{(\delta((\alpha-1)(\gamma+1)(\theta-1) \lambda+\gamma \theta-\gamma+\theta)+(\alpha-1) \lambda \rho)^{2}} .
\end{aligned}
$$

Using equations (25), (26), (29) and (30) we can show that:

$$
\frac{\frac{\partial F}{\partial s_{j}}}{\frac{\partial F}{\partial s_{Y}}}=\frac{1}{\alpha} .
$$

As we assume that

$$
0<\alpha<1, \frac{\frac{\partial F}{\partial s_{j}}}{\frac{\partial F}{\partial s_{Y}}}>1
$$


From equations (31) and (32), we can demonstrate that:

$$
-\frac{\frac{\partial s_{Y}}{\partial \alpha}}{\frac{\partial s_{j}}{\partial \alpha}}=\frac{(\lambda-1)((\gamma+1) \delta(\theta-1)+\rho)}{\delta((\gamma+1)(\theta-1) \lambda+\gamma(-\theta)+\gamma-\theta)+\lambda \rho},
$$

which is smaller than one if and only if

$$
\gamma>\frac{\delta \theta(1-\lambda)+\lambda(\delta-\rho)}{\delta(1-\lambda)+\delta \theta(\lambda-1)}
$$

We can show that

$$
\frac{\delta \theta(1-\lambda)+\lambda(\delta-\rho)}{\delta(1-\lambda)+\delta \theta(\lambda-1)}
$$

is negative. Indeed, the numerator $\delta \theta(1-\lambda)+\lambda(\delta-\rho)$ is positive if and only if

$$
\theta>\frac{\lambda(\delta-\rho)}{\delta(\lambda-1)}>0
$$

Or, we assume that $\theta>1$. The denominator is negative if and only if $\theta>1$ what we assume. Finally, as we assume that $0<\gamma<1$, we always have

$$
\gamma>\frac{\delta \theta(1-\lambda)+\lambda(\delta-\rho)}{\delta(1-\lambda)+\delta \theta(\lambda-1)}
$$

Therefore, the sign of the derivative $\partial y / \partial \alpha$ is positive.

This result points out that competition affects the level of production per capita positively. The explanation of this result can be found in the global effect of competition on the share of human capital devoted to the different sectors. The equilibrium share of human capital devoted to the intermediate goods sector $\left(s_{j}\right)$ depends positively on competition $(\alpha)$. This means that when the degree of competition within the intermediate goods market increases, the aggregate intermediate output and the human capital coming from this sector increase as well. Unlike the equilibrium share of human capital devoted to the intermediate 
goods sector, an increase in competition decreases the equilibrium share of human capital devoted to the final goods sector $\left(s_{Y}\right)$. Indeed, an increase in competition reduces the price of all the intermediate goods and makes it more profitable for the final goods producers to substitute intermediate goods for human capital. By consequence, the demand for this factor decreases. The equilibrium share of human capital devoted to the research sector $\left(s_{n}\right)$ depends negatively on competition. Indeed, an increase in competition leads to a decrease in the market value of research output, reducing R\&D investment. Finally, competition has no effect on the equilibrium share of human capital accumulation $\left(s_{H}\right)$ because the competition does not play any role in the consumer's decision about education. Overall, the positive effect of competition on the equilibrium share of human capital devoted to the intermediate goods sector offsets the negative effect of competition on the equilibrium share of human capital devoted to the final goods sector. This means that competition increases consumer's welfare.

\section{Conclusion}

In this paper, we have presented a generalization of the Bucci's (2003) model in which have disentangled the monopolistic mark-up in the intermediate goods sector, the intermediate goods share in the final output and the returns to specialization in order to have a better measurement of competition. Indeed, unlike BuCCI (2003), in our model, the measure of competition is completely independent of the intermediate goods share in the final output and the returns to specialization. Our main finding is that the result of the Bucci's (2003) model depends critically on the assumption that there is no difference between these three parameters $^{11}$. Indeed, for all values of parameters except $\lambda=\alpha$, we could show that the competition does not play any role in growth. This result is explained by the complementarity of innovation and human capital assumed in the research production. Moreover, we have shown that an increase in competition raises the level of production per capita, increasing consumer's welfare.

In light of these results, one important question remains open on the future research agenda. It would be worth studying how the main result of this paper would change if one assumed that economic growth was simultaneously induced by education investment and $\mathrm{R} \& \mathrm{D}$ activity.

11 We can also note for the interested reader that is also the case for the Bianco's (2009) model. 


\section{Appendix}

In this appendix, we describe the steps followed in order to obtain the main results of this paper. Consider the representative consumer's problem (equations (11) through (13) in the main text). The first order conditions and the transversality conditions are:

$$
\begin{aligned}
& \epsilon_{1}=c^{-\theta} e^{-\rho t}, \\
& -\dot{\epsilon}_{1}=\epsilon_{1} r, \\
& -\dot{\epsilon}_{2}=\epsilon_{1} w u+\epsilon_{2} \delta(1-u), \\
& \epsilon_{1}=\epsilon_{2} \frac{\delta}{w}, \\
& \dot{a}=r a+w u h-c, \\
& \dot{h}=\delta(1-u) h, \\
& \lim _{t \rightarrow \infty} \epsilon_{1 t} a_{t}=0, \\
& \lim _{t \rightarrow \infty} \epsilon_{2 t} h_{t}=0 .
\end{aligned}
$$

Combining equations (35) and (36), we obtain:

$$
\frac{\dot{\epsilon}_{2}}{\epsilon_{2}}=-\delta .
$$

From equation (34), we get:

$$
\frac{\dot{\epsilon}_{1}}{\epsilon_{1}}=-r .
$$

Equation (36) implies that:

$$
\frac{\dot{\epsilon}_{1}}{\epsilon_{1}}=\frac{\dot{\epsilon}_{2}}{\epsilon_{2}}-g_{w} .
$$


Combining equations (41), (42) and (43), we obtain:

$$
r=\delta+g_{w} .
$$

In the balanced growth path equilibrium, the growth rate of the wage accruing to human capital $\left(g_{w}\right)$ is constant (see below). This implies that the real interest rate $(r)$ will be also constant. With a constant real interest rate and using the equation (7), the equation (9) becomes:

$$
V_{n t}=A \lambda(1-\alpha) B^{\lambda} \int_{t}^{\infty} n_{\tau}^{\gamma-1} H_{j \tau}^{\lambda} H_{Y \tau}^{1-\lambda} e^{-r(\tau-t)} d \tau, \tau>t .
$$

In order to compute the market value of one unit of research output at time $t\left(V_{n t}\right)$ along the balanced growth path equilibrium, we use the following equations:

$$
\begin{aligned}
& n_{\tau}=n_{t} e^{g_{n} t}, \\
& H_{j \tau}=H_{j t} e^{g_{H_{j}} t}, \\
& H_{Y \tau}=H_{Y t} e^{g_{H Y} t} .
\end{aligned}
$$

Inserting equations (46), (47) and (48) into equation (45), and after some calculations, we arrive at:

$$
V_{n t}=\frac{A \lambda(1-\alpha) n^{\gamma-1}\left(B H_{j}\right)^{\lambda} H_{Y}^{1-\lambda}}{r-(\gamma-1) g_{n}-(1-\lambda) g_{H_{Y}}-\lambda g_{H_{j}}} .
$$

Such result is obtained under the assumption that

$$
r>(\gamma-1) g_{n}-(1-\lambda) g_{H_{Y}}-\lambda g_{H_{j}} .
$$

In a moment, we will demonstrate that this hypothesis (which assures that $V_{n t}$ is positive for each $t$ ) is always checked along the balanced growth path equilibrium.

Given $V_{n t}$ and making use of equation (10) in the main text, we get:

$$
w_{n}=\frac{C A \lambda(1-\alpha) n^{\gamma-1}\left(B H_{j}\right)^{\lambda} H_{Y}^{1-\lambda}}{\left(r-(\gamma-1) g_{n}-(1-\lambda) g_{H_{Y}}-\lambda g_{H_{j}}\right)} .
$$


From equations (3) and (6), we get the value of the wage rate accruing to human capital employed in the final goods sector:

$$
w_{Y}=(1-\lambda) A H_{Y}^{-\lambda} n^{\gamma} B^{\lambda} H_{j}^{\lambda} .
$$

From equations (2), (5) and (6), we get the value of the wage rate accruing to human capital in the intermediate goods sector:

$$
w=\alpha \lambda A H_{Y}^{1-\lambda} n^{\gamma} B^{\lambda} H_{j}^{\lambda-1} .
$$

Combining equations (33) and (34), we are able to obtain the usual Euler equation, giving the optimal household's consumption path:

$$
g_{c}=\frac{r-\rho}{\theta} .
$$

From the equation above, we clearly see that $r$ must be greater than $\rho$ and at the same time $\theta>0$ in order to have $g_{c}$ positive. From equation (17) and using equation (49), we get:

$$
g_{a}=g_{n}+g_{V_{n}}=g_{n}+(\gamma-1) g_{n}+\lambda g_{H_{j}}+(1-\lambda) g_{H_{Y}} .
$$

Using equations (18), we can rewrite the equation (54) as follows:

$$
g_{a}=(\gamma+1) g_{n} .
$$

From equations (37) and (42), we have:

$$
\frac{\dot{\epsilon}_{1}}{\epsilon_{1}}=-g_{a}+u w \frac{b}{a}-\frac{c}{a} .
$$

Using equations (35) and (36), we get:

$$
\frac{\dot{\epsilon}_{2}}{\epsilon_{2}}=-g_{h}-u \delta .
$$

Equations (18), (50), (51) and (52) together also imply that:

$$
g_{w_{n}}=g_{w_{Y}}=g_{w}=\gamma g_{n} .
$$


From equations (19), (42), (43) and (57), we obtain:

$$
\frac{c}{a}=\delta u+u w \frac{h}{a}
$$

Using equations (18), (55) and (58) and knowing that $u$ is constant at the equilibrium, equation (59) leads to the conclusion that $c / a$ is constant. In other words:

$$
g_{c}=g_{a}=(\gamma+1) g_{n}
$$

Plugging equation (60) into equation (53), we get:

$$
r=\theta(\gamma+1) g_{n}+\rho
$$

Now, equating equations (44), (58) and equation (61), we get the growth rate of $n$ along the balanced growth path equilibrium:

$$
g_{n}=\frac{\delta-\rho}{\gamma(\theta-1)+\theta}
$$

Given $g_{n}$, it is now possible to compute the real interest by using equations (44), (58) and (62):

$$
r=\delta+\frac{\gamma(\delta-\rho)}{\gamma(\theta-1)+\theta}
$$

Combining equations (19) and (62), we get the growth rate:

$$
g_{Y}=g_{c}=g_{a}=(\gamma+1) g_{n}=\frac{(\gamma+1)(\delta-\rho)}{\gamma(\theta-1)+\theta}
$$

From the equations (18) and (62), we obtain the growth rate of human capital:

$$
g_{H}=g_{H_{Y}}=g_{H_{j}}=g_{H_{n}}=g_{n}=\frac{\delta-\rho}{\gamma(\theta-1)+\theta} .
$$

From equations (18), (44), (50), (51) and (58), we get the human capital devoted to the final goods sector: 


$$
H_{Y}=\frac{n \delta(1-\lambda)}{C(1-\alpha) \lambda} .
$$

From equations (18), (44), (50), (52) and (58), we get the human capital devoted to the intermediate goods sector:

$$
H_{j}=\frac{n \alpha \delta}{C(1-\alpha)} .
$$

Combining equations (8) and (62), we get the human capital devoted to the research good sector:

$$
H_{n}=\frac{n(\delta-\rho)}{C(\gamma(\theta-1)+\theta)} .
$$

Combining equations (41), (57) and (63), we obtain the time spending in production:

$$
u^{*}=\frac{(\gamma+1) \delta(\theta-1)+\rho}{\delta(\gamma(\theta-1)+\theta)}
$$

\section{References}

Alvarez-Pelaez, Maria, and Christian Groth (2005), "Too Little or too Much R\&D”, European Economic Reviews, 49(2), pp.437-456.

Bianco, Dominique (2009), "Growth and Competition in a Model of Human Capital Accumulation and Research", Journal of Applied Economic Sciences, 4(3(9) Fall), pp.341-348.

Blackburn, Keith, Victor T. Y. Hung, and Alberto F. Pozzolo (2000), "Research, Development and Human Capital Accumulation", Journal of Macroeconomics, 22(2), pp. 189-206.

Bucci, Alberto (2003), "R\&D, Imperfect Competition and Growth with Human Capital Accumulation”, Scottish Journal of Political Economy, 50(4), pp. 417-439.

Grossman, Gene M., and Elhanan Helpman (1991), Innovation and Growth in the Global Economy, Cambridge, Mass. and London: MIT Press.

Lucas, Robert JR. (1988), "On the Mechanics of Economic Development", Journal of Monetary Economics, 22(1), pp.3-42. 
Romer, Paul M. (1990), "Endogenous Technological Change”, Journal of Political Economy, 98(5), pp.71-102.

\section{SUMMARY}

In this paper, we have presented a generalization of Bucci's (2003) model in which have disentangled the monopolistic mark-up in the intermediate goods sector, the intermediate goods share in the final output and the returns to specialization in order to have a better measurement of competition. Indeed, unlike BuCCI (2003), in our model, the measure of competition is completely independent of the intermediate goods share in the final output and the returns to specialization.

Our main finding is that, unlike BuCCI (2003), we show that the competition does not play any role in growth. This result is explained by the complementarity of innovation and human capital assumed in the research production. Moreover, we have shown that an increase in competition raises the level of production per capita, increasing consumer's welfare. 